\title{
Autonomic Nervous System Response During Scuba Diving Activity
}

\author{
Alberto Hernando ${ }^{1}$, María Dolores Peláez-Coca ${ }^{1,2}$, Carlos Sánchez ${ }^{1,2}$, Juan Bolea ${ }^{1,2}$, David \\ Izquierdo $^{1,3}$, María teresa Lozano ${ }^{1,2}$, Eduardo Gil ${ }^{2,4}$ \\ ${ }^{1}$ Centro Universitario de la Defensa (CUD), Zaragoza, Spain \\ ${ }^{2}$ BSICoS Group, Aragón Institute of Engineering Research (I3A), IIS Aragón, University of \\ Zaragoza, Zaragoza, Spain \\ ${ }^{3}$ Photonic Technologies Group (GTF), Aragón Institute of Engineering Research (I3A), Zaragoza, \\ Spain \\ ${ }^{4}$ Centro de Investigación Biomédica en Red Bioingeniería, Biomateriales y Nanomedicina \\ (CIBER-BBN), Madrid, Spain
}

\begin{abstract}
In this work, Heart Rate Variability indices are computed to analyze the Autonomic Nervous System response during a scuba diving activity. To do that, electrocardiogram signals from 6 subjects were recorded in a reservoir immersion. Two different stages were analyzed: baseline stage, where subjects remained seated outside the water; and immersion stage, where subjects performed physical activity in a non-controlled environment. The smoothed pseudo Wigner-Ville distribution was used for the timefrequency analysis. Results show a statistically significant increase of the mean heart rate and a significant decrease in the root mean square of the successive normal beats during the immersion stage. However, frequencydomain parameters did not show any significant difference between stages. Whereas time-domain parameters reflect a sympathetic activation during the immersion, caused by the predominance of the physical activity and the stress of the challenging environment, frequency-domain parameters are not able to capture it. Further studies are needed to draw more robust conclusions by increasing the number of subjects and exploring alternative methods to compute the Autonomic Nervous System frequency content.
\end{abstract}

\section{Introduction}

Diving is a challenging activity, where there is an increase in hydrostatic pressure proportional to depth (a descent of 10 meters implies a pressure increase of one atmosphere, atm). The immersion has an impact on the diver's body generating an internal response to maintain homeostasis. This response is reflected in the Autonomic Nervous System (ANS) through the balance between its two branches (sympathetic and parasympathetic or vagal). The most common non-invasive technique to measure ANS activity is the analysis of Heart Rate Variability (HRV), extracted from the electrocardiogram (ECG).

The ANS response has been analyzed in several works inside a hyperbaric chamber, which allows an increase of pressure in controlled conditions without going under the water. The results of these studies suggested a reduction in the heart rate and an increase in parasympathetic activity [1-3]. There are only a few studies analyzing ANS response during real immersions and they also show dominance of the parasympathetic activity [4-6]. However, these studies involved lower depths in a pool [5], or they analyzed divers that remained static during the immersion [4].

In this work, a new database with experienced divers performing physical tasks inside the water and surrounded by a challenging non-controlled environment with low visibility is used. A study of the ANS response during this immersion is done in order to see if there is a predominance of the sympathetic activity because of the stress associated to the environment, or a predominance of the vagal activity due to the immersion, or an activation of both branches. To do that, time-domain parameters from the RR series and frequency-domain parameters using the smoothed pseudo Wigner-Ville distribution (SPWVD) are analyzed to see if they could characterize the fast response of the ANS to immersion changes.

\section{Materials}

ECG from 6 experienced SCUBA divers from a diving unit of the Spanish Army (all men), with a mean age of $30.8 \pm 5.9$ years, were registered in a real dive in a reservoir, with the approval of the ethics committee "Comité de ética de la investigación con medicamentos de la in- 
spección general de sanidad de la Defensa". Two different stages were analyzed. In the baseline stage, divers remained seated comfortably in silence and without performing movements during 5 minutes outside the water. In the immersion stage, divers performed a real immersion (between 15 and $25 \mathrm{~m}$ of maximum depth, 49 to $82 \mathrm{ft}$ ) breathing air, developing physical tasks inside the water and surrounded by a challenging environment with low visibility. Three minutes at the maximum depth during the immersion were selected for the analysis of this stage.

Recordings were performed using a Nautilus device [7], which allows the ECG signal to be recorded with three leads at a sampling frequency (fs) of $2000 \mathrm{~Hz}$, along with the atmospheric pressure $(\mathrm{fs}=250 \mathrm{~Hz}$ ).

\section{Methods}

\subsection{ECG Analysis}

A low-pass FIR filter was applied to the ECG signal to remove the baseline interference (cut-off frequency of $0.03 \mathrm{~Hz}$ ) [8]. Heartbeats were detected using a waveletbased algorithm over the second lead of the recorded ECG signal [9]. Ectopic beats and missed and false detections were identified and corrected [10]. As a result, the QRS complexes could be located in the ECG, and the difference between consecutive $\mathrm{R}$ waves was used to generate the RR time series. Then, the time-varying integral pulse frequency modulation model was applied to determine the influence of the ANS on the beat occurrence time series [11]. Using this model, the instantaneous heart rate signal $(H R)$ was created at a sampling rate of $4 \mathrm{~Hz}$.

The mean heart rate $(H R M)$ was obtained by low-pass filtering $H R$ with a cut-off frequency of $0.03 \mathrm{~Hz}$. The heart rate variability signal $(H R V)$ was calculated as the subtraction of these two terms: $H R V=H R-H R M$.

\subsection{Time-Domain Parameters}

In addition to the $H R M$, measured in beats per minute (bpm), three more time-domain parameters could be computed from the beat-to-beat time series [12], obtaining one value per stage as the mean of the 3 or 5 minute-stageduration:

- $S D N N(s)$ : Standard deviation of the Normal-toNormal $(N N)$ intervals, as a measure of statistical dispersion. This parameter could be interpreted as an indicator of overall ANS activity.

- $R M S S D(s)$ : Square root of the mean of the squares of the successive differences between adjacent $N N$ intervals. This parameter mainly reflects the parasympathetic tone. - $p N N 50(\%)$ : Number of pairs of successive $N N s$ that differ by more than $50 \mathrm{~ms}$, divided by the total number of $N N$ intervals.

\subsection{Frequency-Domain parameters}

Time-frequency analysis was applied to $H R V$ to characterize the fast response of the ANS to the immersion. The SPWVD was selected to perform this analysis because it provides better resolution than non-parametric linear methods, independent control of time and frequency filtering, and power estimates with lower variance than parametric methods when rapid changes occur. To attenuate the interference terms, time and frequency smoothing windows were chosen to be Hamming windows of length $2 \mathrm{~N}-1=203$ and $2 \mathrm{~K}+1=513$ samples, respectively [13]

Two main components were considered: a lowfrequency (LF) component, which reflects both sympathetic and parasympathetic activity, and a high-frequency (HF) component, which reflects only parasympathetic activity. As it is well known, parasympathetic component is related to respiratory sinus arrhythmia, so in this work HF band is centred in the respiratory rate, $\operatorname{Fr}$ [13]. Respiratory rate was extracted from the ECG, from QRS slopes and R-wave angle [14]. HF bandwidth is $0.125 \mathrm{~Hz}$, in order to have a similar bandwidth with respect to the LF band. For the 6 subjects, the lower limit of HF band was always above $0.15 \mathrm{~Hz}$ (no overlapping with the LF band).

Four frequency-domain indices are computed during the entire stage, and then one value is given:

- PLF(a.u.): Mean of the power in the LF band [0.04 $0.15 \mathrm{~Hz}]$, in arbitrary units.

- PHF(a.u.): Mean of the power in the HF band [Fr$0.0625-F r+0.0625]$, in arbitrary units.

- PLFn(n.u.): Mean of the instantaneous power in the LF band over the sum of the instantaneous powers of both LF and HF bands. PLFn $=P L F /(P L F+P H F)$, in normalized units.

- $R_{L F / H F}(a d)$ : Mean of the ratio between the instantaneous power in the LF band and the instantaneous power in the HF band. $R_{L F / H F}=P L F / P H F$, adimensional.

Figure 1 shows an example of two time-frequency maps for the same subject, one per stage.

\subsection{Statistical analysis}

Finally, a statistical analysis was performed to compare baseline stage versus immersion stage. First, a ShapiroWilk test was applied to verify whether data distribution is normal. Then, as these distributions were no-normal, the Wilcoxon paired test was applied, with a $p$-value $\leq 0.05$ to define the significance.

\section{Results}

Figure 2 presents the results of time-domain and frequency-domain parameters from baseline and immersion stages during the reservoir immersion. On the one 

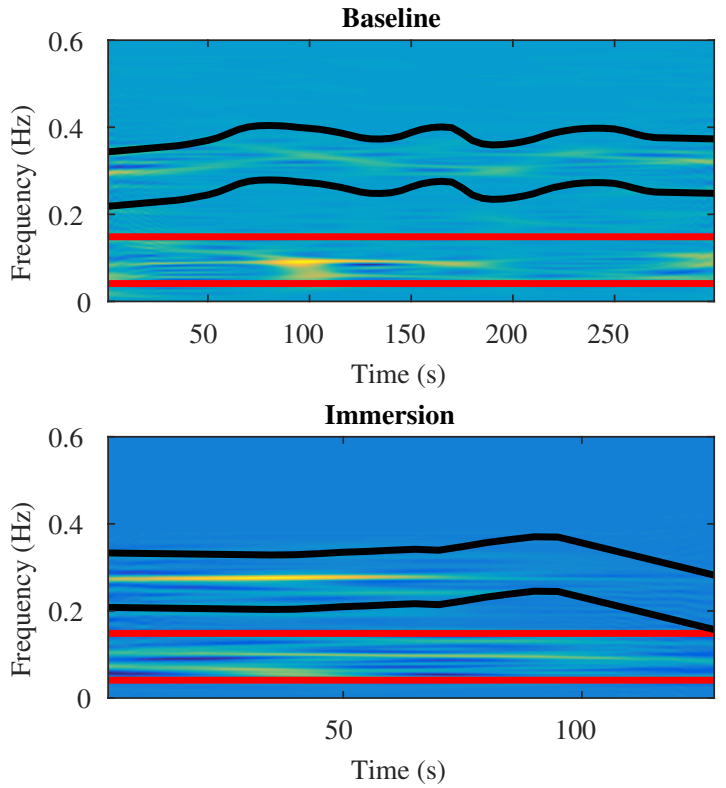

Figure 1. Time-frequency maps for baseline and immersion stages, with the LF band delimited in red and the HF band delimited in black.

hand, time-domain parameters show that $H R M$ significantly increases and $R M S S D$ decreases during the immersion. On the other hand, frequency-domain parameters do not show clear differences between both stages.

\section{Discussion}

The main goal of this work is to study the ANS response during a scuba diving activity while the divers were developing physical tasks inside the water. To that end, an analysis of HRV using time-domain parameters and the SPWVD time-frequency method was performed to characterize the fast response of the ANS to the immersion. A comparison between the two stages (baseline and immersion) is carried out to study how the scuba diving activity affects the ANS response.

Time-domain parameters show a significant increase in $H R M$ and a significant decrease in $R M S S D$. This points out to an activation of the sympathetic system. These results are in contrast with previous works, where a $H R M$ decrease has been reported in hyperbaric chamber [1-3] and in immersions $[4,6]$. This sympathetic activation could be explained by different factors: one could be the predominance of the physical activity and the stress of the challenging environment during the immersion [15]. Another factor is the stress related to the immersion: some studies reported that an immersion performing a stressful task could increase the $H R M$ and the sympathetic activity [5]. The effect of cold water, that also increases the
$H R M$ [16], could be another factor.

On the other hand, frequency-domain parameters do not show any significant differences between the two stages. There is neither a sympathetic activation registered in the $P L F n$ and $R_{L F / H F}$ as reflected in the time-domain parameters, nor a parasympathetic activation registered in the $P H F$ as the bibliography suggests $[1,3,4,6]$. A possible explanation of this is the low number of subjects. In fact, our database is larger ( 15 subjects), but only 6 have been studied because respiratory rate falls into the LF band in the excluded subjects, which could cause an overestimation of the $P L F$ and, consequently, an underestimation of the PHF [17]. In future studies, a new algorithm to extract the frequency-domain parameters that overcome with the respiratory rate limitation and take into account nonlinear relationships of the HRV should be used.

\section{Conclusion}

A study of the ANS response during a scuba diving activity while the divers were developing physical tasks has been performed using time-frequency methods. Timedomain parameters suggest an activation of the sympathetic activity during the immersion. However, frequencydomain parameters do not show any change between baseline and immersion stages. In future studies, the use of alternative methods to compute the ANS frequency content taking into account the intrinsic non-linear dynamics and the influence of respiration is recommended.

\section{Acknowledgements}

This work has been partially financed by Ministerio de Economía, Industria y Competitividad (MINECO) and by fondos FEDER through the project PGC2018-095936-BI00 and RTI2018-097723-B-I00; by Centro Universitario de la Defensa (CUD) under the projects CUD2019-10 and UZCUD2019-TEC-01; and by Aragón Government and European Regional Development Fund through Grupos de Referencia BSICoS (Biomedical Signal Interpretation \& Computational Simulation, T39-20R) and GTF (Grupo de Tecnologías Fotónicas T20-20R). The author would like to thank the assistance as volunteers of the Regimiento de Pontoneros y Especialidades de Ingenieros $n^{\circ} 12$.

\section{References}

[1] V. Lund, J. Laine, T. Laitio, E. Kentala, J. Jalonen, H. Scheinin, "Instantaneous beat-to-beat variability reflects vagal tone during hyperbaric hyperoxia," Undersea Hyperbar. M., vol. 30, pp. 29-36, 2003.

[2] E. Barbosa, J.M. García-Manso, J.M. Martín-González, S. Sarmiento, F.J. Calderón, M.E. Da Silva-Grigoletto, "Effect of Hyperbaric Pressure During Scuba Diving on Autonomic Modulation of the Cardiac Response: Application of 

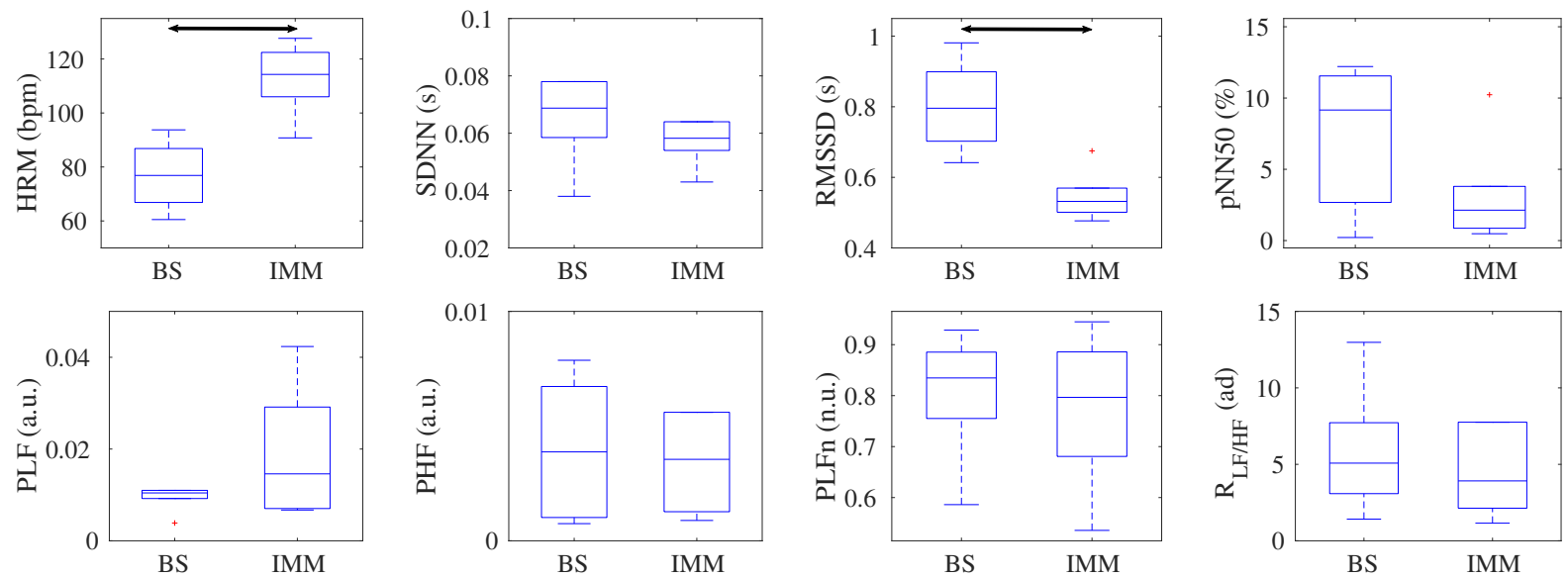

Figure 2. Boxplots of the time-domain and frequency-domain parameters from baseline and immersion stages during the reservoir immersion. Significant differences are represented by a double arrow.

the Continuous Wavelet Transform to the Analysis of Heart Rate Variability," Mil. Med., vol. 175, no. 1, pp. 61-64, 2010.

[3] A. Hernando, M.D. Peláez-Coca, M.T. Lozano, M. Aiger, D. Izquierdo, A. Sánchez, M.I. López-Jurado, I. Moura, J. Fidalgo, J. Lázaro, E. Gil, "Autonomic nervous system measurement in hyperbaric environments using ECG and PPG signals," IEEE J. Biomed. Health Inform., vol. 23, no. 1, pp. 132-142, 2019.

[4] Y. Noh, H.F. Posada-Quintero, Y. Bai, J. White, J.P. Florian, P.R Brink, K.H. Chon, "Effect of Shallow and Deep SCUBA Dives on Heart Rate Variability," Front. Physiol., vol. 9, pp. 110, 2018.

[5] A.D. Flouris, J.M. Scott, "Heart rate variability responses to a psychologically challenging scuba dive," J. Sports Med. Phys. Fitness, vol. 49, n. 4, pp. 382-386, 2009.

[6] F. Chouchou, V. Pichot, M. Garet, J.C. Barthelemy, F. Roche, "Dominance in cardiac parasympathetic activity during real recreational scuba diving," Eur. J. Appl. Physiol., vol. 106, pp. 345-352, 2009.

[7] D. Sokas, M. Gailius, V. Marozas, "Diver physiology monitor and its graphical user interface," Virt. Inst. Biomed., pp. 5-9, 2016.

[8] L. Sornmo, P. Laguna, "Bioelectrical signal processing in cardiac and neurological applications," Elsevier Academic Press, 2005.

[9] J.P. Martínez, R. Almeida, S. Olmos, A.P. Rocha, P. Laguna, "A wavelet-based ECG delineator: evaluation on standard databases," IEEE Trans. Biomed. Eng., vol. 51, no. 4, pp. 570-581, 2004.

[10] J. Mateo, P. Laguna, "Analysis of Heart Rate Variability in the Presence of Ectopic Beats Using the Heart Timing Signal," IEEE Trans. Biomed. Eng., vol. 50, no. 3, pp. 334-343, 2003.

[11] R. Bailón, G. Laouini, C. Grao, M. Orini, P. Laguna, O. Meste, "The Integral Pulse Frequency Modulation with
Time-Varying Threshold: Application to Heart Rate Variability Analysis during Exercise Stress Testing," IEEE Trans. Biomed. Eng., vol. 58, no. 3, pp. 642-652, 2011.

[12] Task Force of the European Society of Cardiology and the North American Society of Pacing and Electrophysiology, "Heart rate variability: standards of measurement, physiological interpretation and clinical use," Circulation, vol. 93, pp. 1043-1065, 1996.

[13] R. Bailón, N. Garatachea, I. de la Iglesia, J.A. Casajús, P. Laguna, "Influence of running stride frequency in heart rate variability analysis during treadmill exercise testing," IEEE Trans. Biomed. Eng., vol. 60, no. 7, pp. 1796-1805, 2013.

[14] J. Lázaro, A. Alcaine, D. Romero, E. Gil, P. Laguna, E. Pueyo, and R. Bailón, "Electrocardiogram Derived Respiratory Rate from QRS Slopes and R-wave Angle," Ann. Biomed. Eng., vol. 40, no. 10, pp. 2072-2083, 2014.

[15] D.R. Seals, D.G. Johnson, R.F. Fregosi, "Hyperoxia lowers sympathetic activity at rest but not during exercise in humans," Am. J. Physiol., vol. 260, pp. R873-878, 1991.

[16] P. Šrámek, M. Šimečková, L. Janský, J. Šavlíková, S. Vybíral, "Human physiological responses to immersion into water of different temperatures," Eur. J. Appl. Physiol., vol. 81, no. 5, pp. 436-442, 2000.

[17] A. Hernando, J. Lázaro, E. Gil, A. Arza, J.M. Garzón, R. López-Antón, C. de la Cámara, P. Laguna, J. Aguiló, R. Bailón, "Inclusion of respiratory frequency information in heart rate variability analysis for stress assessment," IEEE J. Biomed. Health Inform., vol. 20, no. 4, pp. 1016-1025, 2016.

Address for correspondence:

Alberto Hernando Sanz

Centro Universitario de la Defensa (CUD), Academia General Militar (AGM), ctra. Huesca s/n., 50090, Zaragoza, Spain. ahersanz@unizar.es 